\title{
Hypothalamic control of systemic glucose homeostasis: the pancreas connection
}

Macarena Pozo ${ }^{1} \&$ Marc Claret ${ }^{1,2, *}$

${ }^{1}$ Neuronal Control of Metabolism (NeuCoMe) Laboratory, Institut d'Investigacions Biomèdiques August Pi i Sunyer (IDIBAPS), 08036 Barcelona, Spain

${ }^{2}$ CIBER de Diabetes y Enfermedades Metabólicas Asociadas (CIBERDEM), 08036 Barcelona, Spain

*Correspondence: mclaret@clinic.cat (M. Claret)

Keywords: glucose homeostasis, hypothalamus, obesity, diabetes, pancreas

\section{ABSTRACT}

Maintenance of glucose homeostasis is mandatory for organismal survival. It is accomplished by a complex, coordinated interplay between glucose detection mechanisms and multiple effector systems. The brain, in particular homeostatic regions such as the hypothalamus, plays a critical role in orchestrating such a highly integral response. Here we review current understanding of how the hypothalamus senses glucose availability and participates in systemic glucose homeostasis. We provide an update of the relevant signaling pathways and neuronal subsets involved, as well as the mechanisms modulating metabolic processes in peripheral tissues such as liver, skeletal muscle, fat, and especially the pancreas. We also discuss the relevance of these networks in human biology and prevalent metabolic conditions such as diabetes and obesity. 


\section{HISTORICAL ESSENTIALS}

The first evidence that the brain influences glucose metabolism was provided by Claude Bernard in 1849. He observed that stimulation of the base of the fourth ventricle in rabbits caused a dramatic rise in blood glucose. The notion that the brain was the only relevant location for glucoregulation was widely accepted until Banting and colleagues discovered insulin in 1921. These findings heralded an era devoted to investigating the mechanisms of insulin secretion and action in physiology and pathophysiology that left the brain in the background. Nevertheless, in the early 1950s, Jean Mayer proposed the "glucostatic theory", which implied that the brain could detect glucose fluctuations [1], and in the mid-1960s, electrophysiological recordings uncovered glucose-sensing neurons in the hypothalamus [2, 3]. With renewed interest in the brain, the topic of central glucose metabolism control blossomed over the following decades, especially with the advent of powerful techniques and mouse genetics in the 1990s.

\section{HYPOTHALAMIC GLUCOSE-SENSING: THE BASICS}

\section{Location of glucose-sensing neurons}

Glucose is the prime cellular energy source to sustain life and therefore its availability must be constantly monitored. Higher organisms have developed dedicated sensor and effector mechanisms to maintain systemic glucose homeostasis $[4,5]$. The brain uses $\sim 60-70 \%$ of the total glucose. It predominantly expresses high affinity GLUT1 and GLUT-3, allowing effective glucose transport at normal circulating range. Thus the brain does not depend on insulin for glucose uptake, even though insulin is a key signaling molecule.

As the brain is the most avid glucose consumer of the organism, it is teleologically reasonable that the brain executes surveillance functions. Evidence indicates that continuous glucose monitoring is achieved by dedicated cell types located in several regions including the brainstem, corticolimbic areas and hypothalamus (Figure 1A). Notably, neuronal glucose-sensing has received most attention, but the relevance of non-neuronal cells in this biological process has been underscored recently (Box 1). 
While glucose-sensing is a complex, distributed process, extensive research has recognized the hypothalamus as a key nexus of these networks. The hypothalamus is constituted by distinct nuclei morphologically and functionally, including the paraventricular (PVN), ventromedial (VMN), lateral (LH) and arcuate nucleus (ARC), which all contain specific glucose-sensing neuronal populations.

The ARC, which is adjacent to the third ventricle (3V; see Glossary) and the median eminence, contains two antagonistic neuronal populations: orexigenic Agoutirelated peptide (AgRP)-producing neurons and anorexigenic neurons that release Proopiomelanocortin (POMC)-derived peptide $\alpha$-melanocyte-stimulating hormone ( $\alpha$ $\mathrm{MSH}$ ). Together with neurons expressing the melanocortin 4 receptor (MC4R), they constitute the melanocortin system which is essential for appetite, energy expenditure and glucose homeostasis [4]. Competitive binding of $\alpha-M S H$ and AgRP on MC4Rs define the activation magnitude of downstream pathways and effectors. AgRP neurons, which send inhibitory Gamma-aminobutyric acid (GABA) terminals onto POMC neurons, also contain the orexigenic neuropeptide $Y$ (NPY) [4]. The overall balance between orexigenic and anorexigenic forces defines the final metabolic outcome. AgRP neurons are considered glucose-inhibited (GI) cells, while most studies suggest that POMC neurons are glucose-excited (GE) [5]. However, further studies are warranted as recent reports raise the possibility that modulation of POMC neuron activity by glucose is due to presynaptic inputs rather than direct detection [6].

ARC POMC and AgRP neurons project to numerous extra-hypothalamic and hypothalamic regions, including the VMN [7]. A subset of VMN neurons sense changes in extracellular glucose concentration, including both $\mathrm{GE}$ and $\mathrm{GI}$ neurons $[5,8]$. Steroidogenic factor 1 (SF1) expressing neurons are particularly abundant and express receptors for key metabolic hormones, but only $\sim 10 \%$ are glucose-responsive [8]. In addition to regulating energy balance, the $\mathrm{VMN}$ is critical to initiate the counterregulatory response to hypoglycemia [9].

The LH consists of a heterogeneous structure of neuronal populations, including the well-described orexin and melanin-concentrating hormone (MCH) neurons, amongst other neuronal types. In general terms, a portion of orexin neurons are GI, while subsets of $\mathrm{MCH}$ neurons are GE [5]. This area is implicated in arousal, appetite and reward. 


\section{Molecular mechanisms of glucose-sensing}

The molecular foundations underlying neuronal glucose-sensing are diverse and not completely understood, but plausibly similar to classical mechanisms operating in pancreatic $\beta$-cells. Based on their electrical response to changes in extracellular glucose levels, glucose-sensing neurons are classified as GE or GI [5]. Glucose uptake by GE neurons seems to be mediated by heterogeneous mechanisms, as differential expression of glucose transporters (mainly GLUT-2, GLUT-3, GLUT-4 and SGLT) has been reported depending on their neuropeptide identity or localization. Generally speaking, depolarization in GE neurons operates similarly to pancreatic $\beta$-cells: via glucokinase (GK) and ATP-mediated closure of $K_{\text {ATP }}$ channels (Figure 1B). [10, 11]. GK has a low glucose affinity, so its activity varies substantially and proportionally to glucose concentration. Given these distinctive features, GK is considered a critical element and has been used to predict glucose-sensing populations of neurons [5]. The cellular energy sensor AMPK has also been proposed to play a relevant role in glucosesensing, [12].

Similarly, subsets of GI neurons express the same glucose transporters [5]. A large proportion of GI neurons seem to rely on GK [11] but exceptions are reported, which suggests that it is not essential for glucose-sensing [13]. In the VMN, an important region for hypoglycemia detection, low glucose levels depolarize GI neurons via closure of chloride channel through a mechanism mediated by AMPK and nitric oxide (Figure 1B) [5]. In addition to GI neurons that respond to fluctuations in glucose via its metabolization, the activity of some GI neurons is modulated by the glucose molecule itself. This is the case of orexin-expressing $\mathrm{Gl}$ neurons in the $\mathrm{LH}$, which can respond to either glucose or 2-deoxyglucose (2-DG), although the precise mechanisms are not fully understood [6]. The diversity of brain regions, neuronal types and molecular mechanisms involved in glucose-sensing underscores the complexity and heterogeneity of this fundamental biological process and suggests the existence of intricate neurocircuits (Box 2). According to these inputs, the hypothalamus conveys multiple effector mechanisms to precisely adjust the metabolic output of peripheral tissues thus preserving glucose homeostasis. These effects involve the main metabolic tissues, including the liver, skeletal muscle, adipose tissue and pancreas. The main focus of this review is the pancreas. 


\section{HYPOTHALAMIC CONTROL OF HEPATIC GLUCOSE PRODUCTION}

Hepatic glucose production (HGP) is one of the major processes implicated in systemic glucose homeostasis. Ample evidence indicates that the hypothalamic action of metabolic hormones (insulin and leptin) and nutrients influence HGP in rodents [14]. Hypothalamic leptin, preferentially via POMC neurons, suppresses HGP and increases insulin-independent tissue glucose uptake [14]. The beneficial effects of hypothalamic leptin action on HGP are also apparent in pathophysiological conditions such as dietinduced insulin resistance [15] or severe insulin deficiency (Box 3).

The physiological role of insulin signaling in the hypothalamus is more controversial. Several pharmacological and genetic studies suggest that hypothalamic insulin suppresses HGP in rodents via neuronal KATP channels, PI3K and the vagus nerve [14]. Surprisingly, direct hepatic insulin signaling seems to be dispensable for suppression of glucose output in mice if the central mechanisms remain functional [16]. In dogs, insulin also engages brain pathways and liver changes in glucoregulatory genes but without significant effects on HGP [17]. These findings call into question a physiological role for central insulin upon HGP control, in favor of direct insulin effects in liver. This controversy may partly arise from technical issues and differences in glucose physiology between species.

Collectively, evidence suggests that adequate HGP is achieved by the complex coordination of both liver and central-mediated indirect effects. It is likely that the hypothalamus, through integrated sensing of circulating hormones and nutrients, finetunes hepatic glucose output according to metabolic status. An extensive discussion can be found elsewhere $[18,19]$

\section{HYPOTHALAMIC CONTROL OF GLUCOSE METABOLISM IN SKELETAL MUSCLE}

Skeletal muscle is the major site for glucose disposal, but current understanding of underlying hypothalamic mechanisms is scarce. Intracerebroventricular (i.c.v.) insulin augments muscle glycogen synthesis and this is prevented by glucose co-infusion [20]. Leptin microinjection in the VMN, but not in other hypothalamic nuclei, preferentially increases glucose uptake in skeletal muscle and heart [21, 22]. These effects are mediated via the melanocortin system: VMN leptin action is abolished by MCR 
antagonists, while delivery of MCR agonists, either into the VMN or i.c.v., is sufficient to increase muscle glucose uptake [23].

The advent of optogenetic and chemogenetic strategies has allowed for more accurate functional studies. Chemogenetic activation of VMN SF1 neurons increases whole-body insulin sensitivity and the uptake of glucose in red-type skeletal muscle and heart under hyperinsulinemic-euglycemic conditions. Parallel stimulation of insulin signaling in soleus muscle is observed after activation of VMN SF1 neurons during the clamp period [24]. These studies confirm previous findings on the role of the VMN upon insulin-induced glucose uptake in muscle and heart.

Orexin administration into the VMN also promotes insulin-induced glucose uptake and glycogen synthesis in skeletal muscle but not in WAT. This is part of a nonhomeostatic system, involving orexin neurons in the $\mathrm{LH}$, required to support glucose metabolism in motivated behaviors [25].

\section{HYPOTHALAMIC CONTROL OF GLUCOSE METABOLISM IN ADIPOSE TISSUE}

Glucose uptake in white adipose tissue (WAT) is largely dependent on insulin action, although it only accounts for $\sim 5-10 \%$ of total. However, WAT supplies gluconeogenic precursors (glycerol and non-esterified free fatty acids) for HGP, thus efficient control of lipolysis contributes to coordinating glycemia and adiposity.

Evidence suggests that lipid mobilization is divergently regulated by hypothalamic insulin and leptin. Infusion of insulin into the mediobasal hypothalamus of rodents suppresses sympathetic outflow to WAT, thereby restraining lipolysis. It also induces de novo lipogenesis by modulating the expression of key lipogenic enzymes [26]. Intra-hypothalamic insulin administration combined with clamp experiments revealed that glycerol correlated with HGP, while neither of these parameters correlated with circulating insulin levels [26]. Conversely, i.c.v. leptin reduces WAT lipid storage by increasing lipolysis and inhibiting lipogenesis. The latter process is mediated by PI3K and sympathetic outflow [27].

The melanocortin system is a critical modulator of WAT metabolism. Central injection of MC3/4R agonist in rodents enhances the sympathetic drive to specific WAT depots and increases lipolysis-related genes [28]. In contrast, pharmacological or genetic disruption of MC4R markedly promotes lipid uptake, triglyceride synthesis, and 
fat accumulation. This is associated with improved insulin sensitivity and glucose uptake in WAT. Importantly, these effects are irrespective of food intake and anticipate adiposity changes [29].

Brown adipose tissue (BAT) is highly flexible in terms of glucose uptake potential and can significantly contribute to glucose uptake and thus whole-body glucose metabolism under certain conditions. VMN leptin delivery or chemogenetic activation of SF1 neurons stimulates BAT glucose uptake $[21,22,24]$. In the LH, selective restoration of MC4R expression in MC4R-null mice increases the sympathetic outflow to BAT, associated with GLUT4 upregulation and effective glucose absorption in this tissue [30]. Elegant optogenetic studies revealed that acute stimulation of ARC AgRP neurons deteriorates systemic glucose metabolism through impairment of insulin-stimulated glucose uptake into BAT. This is due to gene expression reprogramming of BAT towards a myogenic signature [31].

\section{HYPOTHALAMIC CONTROL OF PANCREATIC FUNCTION}

The principal role of the endocrine pancreas, represented by the islet of Langerhans, is to maintain systemic glucose homeostasis. It has been known for decades that the brain influences pancreatic islet physiology via sympathetic and parasympathetic inputs, thus fine-tuning endocrine functions according to metabolic needs. However, our understanding of the neurocircuits and molecular mechanisms involved is still rudimentary. This section summarizes current knowledge of hypothalamic control of insulin and glucagon release, as prominent pancreatic hormones with critical roles in maintaining glucose levels within physiological range (Figure 2).

\section{Brain-pancreas connection: neuroanatomical, neurochemistry and neuropharmacology studies}

\section{Neurocircuits}

Virus-assisted Cre-mediated retrograde tracing strategies have established that the main route originates in the hypothalamus (ARC, VMN and LH) and communicates to the brainstem (nucleus tractus solitarius and dorsal motor nucleus of the vagus) via the $\mathrm{PVN}$, the raphe $(\mathrm{Ra}), \mathrm{A} 5 / \mathrm{C} 1$ cell groups in the pons, and the periaqueductal gray ventral 
zone (PAG) in the midbrain. Brainstem structures innervate pancreatic islets via the vagus and/or spinal cord (Figure 2) [32]. This study thus defines an in vivo map of the neuronal networks, emphasizing the hypothalamus as a critical hub linking with the endocrine pancreas.

\section{Neuropharmacology and neurochemistry}

The role of central glucose or insulin in pancreatic function regulation is controversial. Intracerebroventricular administration of glucose, and subsequent glucose-stimulated insulin secretion (GSIS) assessment in rats, improves glucose handling and increases plasma insulin levels. This observation cannot be attributed to higher systemic glucose levels [33]. However, a similar approach shows a K circulating insulin levels [34]. Regarding insulin, studies in dogs concluded that ventricular injection of this hormone stimulates pancreatic insulin secretion under constant baseline concentration of glucose [35]. Likewise, cerebral insulin causes a fast increase in plasma insulin and a concomitant decrease in glucose levels in rats via $\mathrm{K}_{\text {ATP }}$ channels [36]. However, other studies failed to confirm these findings [37, Ishihara 2009 \#12091]. These discrepancies, which may be of methodological origin, lead to some considerations regarding the overall interpretation and physiological relevance of results. Factors include the divergent results using different experimental species, the use of suprapharmacological concentrations of the hormone and non-physiological delivery routes, and the fact that i.c.v. insulin actions are not restricted to the hypothalamus, and thus other brain areas may be reached.

It has also been suggested that classical hypothalamic neuropeptides play a role in endocrine pancreas modulation. Acute central delivery of MC4R agonists in mice cause a potent reduction in plasma insulin levels, which is not secondary to a lowering of blood glucose or food intake [38]. Consistently, mice with genetic loss or overexpression of melanocortin signaling exhibit changes in circulating insulin, irrespective of body weight and feeding $[38,39]$. However, other studies show that central effects of MC4R agonists/antagonists on insulin levels are absent or are secondary to food intake changes [40-42]. Thus, the central melanocortin system's role in insulin secretion remains debatable, and divergent results may be the consequence of diverse methodological issues and/or compensatory mechanisms in chronic models. 
Evidence also suggests a stimulatory effect of central NPY on insulin release. Ventricular delivery of NPY promotes insulin secretion and potentiates the insulinemic response to hyperglycemia, independent of food intake [43]. In line with this, central subchronic administration of AgRP results in increased insulin concentrations independent of hyperphagia. However, as plasma glucose was not assessed, changes in insulin could be secondary [44].

\section{Arcuate nucleus of the hypothalamus}

\section{Glucose-sensing mechanisms}

GK allows for glucose sensing within physiological range and, in the brain, it is highly expressed in ARC relative to other areas [32]. As the GK/Hexokinase-1 (HK1) ratio activity is critical for glucose-sensing, viral-mediated overexpression of HK1 is predicted to alter this process. HK1 overexpression in the ARC rendered animals glucose intolerant likely due to reduced glucose-stimulated insulin secretion (GSIS) [32]. These results suggest that glucose-sensing in the ARC influences pancreatic endocrine function.

\section{Melanocortin neurons}

Studies aimed at understanding the role of mitochondrial dynamics, as a bioenergetic adaptation to the fluctuations in nutrient availability, uncovered a link between POMC neurons and endocrine pancreas [45]. Deletion of the mitochondrial fusion protein Mitofusin 1 (Mfn1) in POMC neurons of mice results in glucose intolerance despite normal insulin sensitivity without energy balance repercussions. These glucose metabolism perturbations are due to impaired insulin release in response to glucose. Pharmacological rescue studies showed that enhanced sympathetic function and excessive reactive oxygen species (ROS) production underlie abnormal GSIS in these mice [45].

Impaired melanocortin system development in mice also impacts on pancreatic function [46]. High-fat diet administration to lactating mothers leads to aberrant innervation of POMC neuron axons to several hypothalamic areas and parasympathetic fibers to pancreas in the offspring. This is associated with altered 
GSIS. Interestingly, genetic loss of insulin receptor in POMC neurons protected offspring from abnormal neuronal innervation and insulin release [46].

Chemogenetic activation of AgRP neurons acutely induces peripheral insulin resistance and increases plasma insulin within $1 \mathrm{~h}$ without changes in glucagon or corticosterone levels [31]. However, enhanced insulin release may be a compensatory mechanism to counteract insulin resistance rather than a direct effect. In contrast, no effects on glucose metabolism are found when neighboring POMC neurons are chemogenetically activated.

Collectivelly the current data suggest that while SF1 VMN neurons are critical for counterregulation, hypothalamic neurons may be important for fine-tuning postprandial insulin release (Figure 2).

\section{Ventromedial nucleus of the hypothalamus (VMH)}

\section{Counterregulatory response}

Classical VMN lesion and stimulation studies suggested a direct neural influence on pancreatic function $[47,48]$. In particular, the VMN is important for hypoglycemia detection and counterregulatory response $[49,50]$ (Figure 2). Low glucose levels in the VMN depolarize GI neurons via closure of chloride channel through a mechanism mediated by AMPK and nitric oxide [5]. Indeed, pharmacological activation of VMNAMPK enhances the counterregulatory response, while its genetic inhibition results in the opposite effect $[51,52]$. Furthermore, hypoglycemia-induced glucagon release is blocked by the administration of glucose or K KTP channel inhibitors into the VMN [53]. Consistently, intra-VMN insulin exerts an inhibitory effect on $\alpha$-cell glucagon release [54].

Glucose-sensing determinants in the VMN also impact on pancreatic function. In a set of elegant studies, remote activation of GK-expressing VMN neurons increases plasma glucagon and lowers insulin levels, while inhibition of VMN neurons reduces blood glucose, raises insulin levels and suppresses feeding [55]. Genetic loss of GK in SF1 neurons causes a sex-dependent phenotype only in females, characterized by fat accumulation, impaired hypoglycemia-induced glucagon secretion, and lack of autonomic nervous system activation by neuroglucopenia. This gender dimorphism 
suggests an interaction between glucose sensing by GK and sex-hormone signaling [56].

In mouse VMN neurons, absence of functional KATP channels, which are also critical for glucose-sensing, causes severe glucagon secretion deficit under hypoglycemia or neuroglucopenia conditions. However, ex vivo islets showed normal $\alpha$-cell function in mutant animals, which indicates the importance of central KATP channels in modulating glucagon responses [57]. GABA may mediate these effects, as pharmacological manipulation of $\mathrm{K}_{\text {ATP }}$ channels in the VMN results in altered GABA release within this region, concomitant with changes in glucagon-mediated hypoglycemia responses [58]. Consistently, increased GABAergic tone in VMN suppresses counterregulatory responses to a hypoglycemic stimulus [59].

Effective glucoregulation seems to be achieved via glutamatergic SF1 neuronal subsets. Genetic disruption of glutamatergic transmission in SF1 neurons does not alter basal body weight, although it does predispose mice to gain weight when fed with a Western diet. Notably, the counterregulatory response to insulin-induced hypoglycemia and central glucopenia is impaired in mutant mice [60].

Optogenetic and chemogenetic approaches can be used to investigate causality between cellular changes and functional outcomes. Direct photoinhibition of SF1 neurons in mice does not alter fasting blood glucose concentration, but dramatically impairs the ability to recover from insulin-induced hypoglycemia by blocking the increase in glucagon and corticosterone [61]. Conversely, activation of SF1 neurons causes a diabetogenic-like effect characterized by hyperglycemia and glucose intolerance. This hyperglycemic response is partially mediated by the combination of GSIS inhibition and enhanced glucagon release. Neurocircuit mapping studies identified SF1 neurons as part of an ascending glucoregulatory circuit that also includes extra-hypothalamic regions [61].

\section{Hormone signaling pathways}

Insulin delivery into the VMN exerts inhibitory effects on glucagon release under both normoglycemic and hypoglycemic conditions [54]. Indeed, electrophysiology recordings showed that insulin hyperpolarizes SF1 neurons through activation of KATP channels [62]. However, genetic inhibition of insulin signaling in these neurons does 
not cause any obvious pancreatic phenotype [62]. These divergences may be the result of compensations in the genetic constitutive model when compared to acute pharmacological studies. Notably, leptin signaling in the VMN does not seem to impact on pancreatic function $[21,22,63]$.

\section{EVIDENCE FOR CENTRAL CONTROL OF GLUCOSE HOMEOSTASIS IN HUMANS}

Substantial evidence in rodents supports the notion that the hypothalamus plays a role in systemic glucose homeostasis. However, given the artificial nature of the experimental strategies used (suprapharmacology, genetic modifications, opto- and chemogenetics), the real contribution of brain mechanisms to physiological glucose control is controversial. This discussion is especially relevant in the context of human biology, but in this regard obvious methodological difficulties arise.

Indirect evidence of central control of glucose metabolism is backed by expression of glucose-sensing determinants and metabolic hormone receptors in the human hypothalamus [64]. Furthermore, functional imaging techniques have shown that the hypothalamus, and other brain areas, respond to glucose and insulin $[65,66]$.

To explore the role of brain insulin signaling in a selective non-invasive manner, intranasal insulin (INI) has been used in clinical studies. INI delivery transiently increases insulin concentration in the cerebrospinal fluid and influences the activity of various brain regions. The reported effects of INI are diverse, including reduction of appetite and body weight [67], suppression of systemic lipolysis [68], and improved hepatic energy metabolism [69]. In terms of glucoregulation, INI decreases postprandial insulin levels, and hyperinsulinemic-euglycemic clamp studies revealed enhanced whole-body insulin sensitivity [70, 71]. These effects are positively associated with increased parasympathetic activity and hypothalamic activation assessed by imaging techniques [71]. The improvement in insulin sensitivity may be due to HGP suppression and stimulation of glucose uptake after INI delivery [72]. Although INI primarily impacts the brain [73], it should be considered that minor transient systemic insulin absorption could theoretically mediate part of the effects attributed to the brain.

Another reported strategy to investigate central glucoregulation in humans is by investigating the effects of diazoxide. To examine its extra-pancreatic effects, 
Kishore and collaborators conducted pancreatic clamps in healthy humans. Diazoxide causes a $\sim 30 \%$ reduction in glucose production without changes in uptake. Although this study does not directly prove that these effects are mediated by brain $\mathrm{K}_{\text {ATP }}$ channel modulation, complementary rodent studies do support this notion [74].

These studies suggest that the brain, and in particular the hypothalamus via insulin signaling, modulates peripheral glucose metabolism in healthy humans. Importantly, several reports also show a lack of glucoregulation effects after INI or diazoxide treatment in obese or type-2 diabetic (T2D) patients $[71,72,75]$. This, together with imaging studies reporting selective impairment of insulin signaling in the hypothalamus and other areas [76], supports the concept of brain insulin resistance. Despite the manifest importance of the brain's insulin signaling in glucose homeostasis control, it has yet to be demonstrated whether central defects causally contribute to metabolic dysfunction in humans.

\section{CONCLUDING REMARKS}

The brain controls many essential homeostatic functions, so it seems implausible that glucose metabolism is entirely regulated by a peripheral mechanism. In recent years, major advances in mouse genetics and experimental technology have substantially consolidated the pioneering concept that suggests a role for the brain in peripheral control of glucose metabolism. Extensive literature posits the hypothalamus as an important glucoregulatory center, and there is little doubt that it can influence aspects of glucose homeostasis in response to nutrients, hormones, neuropeptides and stress situations such as hypoglycemia. However, experimental controversy and uncertainty about the role of the hypothalamus in the physiological control of daily glucose metabolism have led to skepticism. Further studies need to unequivocally address

these questions and strictly control the metabolic context in which the study is performed (see Outstanding Questions).

Despite these flaws, a basic conceptual model of glucoregulation suggests the existence of complex, coordinated interactions between the brain and peripheral tissues [77]. Under hypoglycemic conditions, the brain (and in particular the VMN) plays a key role in orchestrating the glucoregulatory response. Under normal conditions, the brain would exert fine adjustments to diverse peripheral responses to 
achieve glucose homeostasis. However, defective central mechanisms would cause mild glucose metabolism perturbations that could be compensated peripherally. Similarly, altered peripheral pathways could be counterbalanced by the brain. Therefore, it seems plausible that multiple defects in regulatory systems may be required for diabetes development [77]. Future research will be fundamental to delineate the central machinery underlying glucose homeostasis and to design novel therapeutics for metabolic disorders such as obesity and T2D.

\section{ACKNOWLEDGEMENTS}

We would like to thank Servier Medical Art for figure graphics. This work was supported by the European Research Council (ERC) under the European Union's Horizon 2020 research and innovation programme (grant agreement 725004) and CERCA Programme/Generalitat de Catalunya. M.C. is a recipient of a Miguel Servet 2 contract (MSII15/00025) from Instituto de Salud Carlos III, and co-financed by the European Regional Development Fund (ERDF), "A Way to Achieve Europe". This work was carried out at Esther Koplowitz Centre. 


\section{BOXES:}

\section{Box 1. Non-neuronal brain glucose-sensing}

\section{Tanycytes}

Tanycytes are ependymal cells surrounding the ventricular walls and the floor of the third ventricle of the brain. These specialized cells, and not neurons, are in direct contact with cerebrospinal fluid and thus they are ideally positioned to sense glucose fluctuations. Recent evidence supports the notion that tanycytes exhibit glucosesensing properties. For example, they express critical determinants of glucose-sensing such as GLUT2, GK and KaTP channels [78]. Notably, in primary cultures of tanycytes and brain slices, an increase in extracellular glucose evokes an ATP-mediated rise in intracellular $\mathrm{Ca}^{2+}$. The underlying mechanisms remain largely unknown, but glucose could act via G-protein-coupled receptors to directly trigger intracellular $\mathrm{Ca}^{2+}$ mobilization and transfer the signal to neighboring cells [78]. Collectively, these evidences indicate that tanycytes exhibit multiple mechanisms to respond to glucose fluctuations. However, the in vivo relevance of tanycytes glucose-sensing and their participation in the global integration and regulation of systemic glucose homeostasis remains unknown.

\section{Astrocytes}

Astrocytes play a critical role in fueling neurons via lactate production, and hypothalamic glucose-sensing has been shown to be altered when astrocyte-to-neuron fueling is disrupted. For example, pharmacological blockage of astroglial glutamate metabolism blunts neuronal activation [79]. In addition to modulate neuronal glucosesensing indirectly, astrocytes also exert direct glucoregulation via glucose transporters, as changes in glial GLUT1 in the hypothalamus mediate glucose-sensing in this region and influence glucose production [80]. Furthermore, global inactivation of GLUT2 gene, which is preferentially expressed in astrocytes, causes abnormal glucagon secretion upon hypoglycemia. However, selective re-expression of GLUT2 in glia (but not in neurons) restores hypoglycemia-induced glucagon release [81]. More recently, Garcia-Cáceres and colleagues have unveiled the relevance of astroglyal insulin signaling in brain glucose uptake and in response to changes in systemic glucose availability. Genetic ablation of insulin receptor in astrocytes impairs glucose-induced 
POMC neuron activation and the physiological responses to fasting or glucoprivation [82]. Together, these results emphasize the role of astrocytes upon glucose-sensing and homeostasis and suggest that astroglial networks form an intricate sensing unit with neurons.

\section{Box 2. Brain circuits integrating glucose-sensing}

The hypothalamus is a prominent glucose-sensing area implicated in glucoregulation. However, it is now recognized that this biological process is in fact mediated by a complex and distributed neuroregulatory network that includes extra-hypothalamic nuclei. Glucose-sensing neurons have been described in brainstem structures such as the dorsal motor nucleus of the vagus (DMV), nucleus tractus solitarius (NTS) and the parabrachial nucleus (PBN). While the DMV and NTS contain both GE and GI neurons, the PBN mainly include GI neurons consistent with a role in the counterregulatory response [83]. Furthermore, glucose-sensing neurons have also been reported to exist in corticolimbic areas including the nucleus accumbens (NAC) and amygdala [84]. Within the NAC, GE neurons are mainly found in the shell whereas $\mathrm{GI}$ are predominantly localized in the core region. Similarly, the amygdale also contains GE and GI neurons that express GK and respond to intragastric glucose administration and glucoprivation induced by 2-DG [84]. Nevertheless, the role of glucose-sensing in the reward system, its influence on behavior and implication in physiology/pathophysiology remains to be elucidated.

The distributed anatomy of glucose-sensing neurons amongst several brain regions strongly suggest the existence of sophisticated interactions to integrate and respond adequately to glucose fluctuations. A precise delineation of these neurocircuits is just starting to emerge thanks to the implementation of optogenetic methodologies. In this regard, a discrete neurocircuit implicated in hypoglycemia response has been recently unveiled in a set of elegant studies. This circuit involves a subset of neurons of the lateral PBN (LPBN), that co-express leptin receptor and cholecystokinin, which are activated by glucoprivic stimuli [85]. Optogenetic stimulation of these neurons promotes a counterregulatory-like response via downstream SF1 neurons of the VMN. Further studies have shown that, amongst the different brain areas that VMN SF1 neurons project, only the bed nucleus of the stria 
terminalis (BNST) mediates the response to hypoglycemia [61]. Collectively, these studies offer compelling evidence of an ascending glucorregulatory circuit involving LPBN $\rightarrow$ VMN-SF1 neurons $\rightarrow$ BNST.

In contrast, the neuroanatomy of circuits implicated in glucose homeostasis remains largely unknown. It is well described that the ARC is interconnected with the VMN, and also sends projections to the LH and the PVN. These three nuclei are synaptically connected with preganglionic parasympathetic neurons of the intermediolateral cell column (IML) [86]. These anatomical connections suggest potential circuits and effector pathways to peripheral tissues thus influencing glucose homeostasis [87].

An intriguing unsolved question is how these apparently distinct neurocircuits involved in glucose counterregulation, homeostasis and reward are able to cross-talk and coordinate appropriate responses. It seems plausible that adequate sensing and subsequent adjustments to maintain euglycemia is achieved by a precise communication amongst these different levels of control. Understanding this complexity is a challenge for years to come, but a critical aspect to design better therapies for diabetes.

\section{Box 3. Life without insulin}

The long-standing dogma that life without insulin is not possible has been challenged recently. Shortly after the identification of leptin by Dr. Jeffrey M Friedman and colleagues in 1994 [88], various teams reported that leptin treatment could improve glucose metabolism in rodent models $[89,90]$. These effects appeared to be centrally mediated, as i.c.v. delivery of leptin normalized glucose levels independent of appetite in diabetic models [91]. Further evidence for the hypothalamic effects of leptin as an antidiabetic agent was provided via elegant virogenetic reactivable technology. Reexpression of leptin receptors (LepR) in the ARC of mice globally lacking LepR improved hyperinsulinemia and diabetes with modest effects on body weight [92]. While these effects seemed to be the consequence of insulin-sensitizing effects of leptin, studies also described insulin-independent mechanisms [93].

The first attempt to directly address whether insulin was dispensable for glucoregulation was conducted by Dr. Roger Unger's group. In their study, adenoviral- 
mediated systemic leptin overexpression ameliorated hyperglycemia and blocked body weight loss in autoimmune and chemically-induced rodent models of diabetes. Notably, this metabolic improvement was observed in all the studied animals within 10 days of treatment in the context of near-complete insulin deficiency [94]. Subsequent studies reported that these effects were indeed centrally-mediated. I.c.v. administration of leptin reversed lethality, hyperglycemia and other associated alterations in mice with undetectable circulating insulin levels [95]. To make sure that insulin traces were not underlying the beneficial effects of leptin, the authors developed a model of complete insulin loss by diphtheria toxin-induced $\beta$-cell death. Loss of function and re-expression experiments revealed a neurocircuitry whereby leptin engages hypothalamic GABA and POMC neurons to normalize the metabolic aberrations caused by insulinopenia [96]. Such beneficial effects have been attributed to a variety of mechanisms, including suppression of hyperglucagonemia or glucagon responsiveness, enhanced glucose uptake by brown adipose tissue, and muscle as well as improved liver metabolism [94-96]. Collectively, these studies demonstrate the existence of neurocircuits and molecular mechanisms that can be engaged to normalize glucose metabolism in the context of insulin absence. The underpinnings and the relevance of these circuits in normal physiology are still unknown, but they may be useful to exploit therapeutic opportunities. 


\section{GLOSSARY:}

Chemogenetics: technology that in neuroscience is used to remotely modulate the activity of specific neuronal subsets in freely moving animals. It is based on the use of "designer receptors exclusively activated by designer drugs" (DREADDS) that are activated by otherwise inert compounds. It is an alternative to optogenetics, which allows for long timescale interventions.

Diazoxide: commercially-available drug which is used in clinical practice. It acts as a $\mathrm{K}_{\text {ATP }}$ channel activator, markedly inhibiting insulin release via hyperpolarization of the cell membrane and a subsequent decrease in calcium influx.

Depolarization: rapid shift of cell membrane resting potential, becoming temporarily less negative. This is achieved via opening of channels allowing positive ions to flow into the cell.

Hyperinsulinemic-euglycemic clamp: gold-standard method to quantify insulin sensitivity in vivo. It consists of continuous insulin infusion to achieve a pre-established insulin concentration (hyperinsulinemic). Simultaneously, glucose is intravenously administered at a variable rate to maintain euglycemia. The steady-state rate of glucose infusion is directly correlated with insulin sensitivity. Combined incorporation of radioactive tracers allows for specific tissue measurements.

Glucose counterregulatory response: adaptive and coordinated defensive process aimed at restoring euglycemia when hypoglycemia occurs. It is mainly characterized by i) reduced insulin secretion; ii) increased glucagon release; iii) increased adrenomedullary epinephrine; iv) enhanced appetite.

Hyperpolarization: the opposite of depolarization.

Islet of Langerhans: organizational and functional regions of the pancreas that contain and release endocrine hormones. They are predominantly formed by insulin-secreting $\beta$-cells ( 70\%) and glucagon-secreting $\alpha$-cells ( 20\%).

Intracerebroventricular: invasive surgery procedure that allows for direct injection of substances into the cerebral ventricles.

Mitochondrial fusion: dynamic biological process by which two mitochondria merge into one single compartment. It is mediated by complex enzymatic machinery that assists in the process of inner and outer mitochondrial membrane fusion. 
Optogenetics: technology that uses optic and genetic tools to modulate the activity of targeted neurons in vivo. This strategy consists in the genetic modification of neurons to express light-sensitive ion channels. Neuron activity can be modulated by illumination with different light sources.

Pancreatic clamp: experimental technique in which somatostatin is infused to inhibit endogenous insulin, glucagon and growth hormone secretion. Suppressed hormones are usually replaced by controlled intravenous administration, and the targeted clamp levels for glucose are maintained by varying rates of glucose or insulin.

Third ventricle: one of the four cerebrospinal fluid-filled cavities that constitute the brain ventricular system. It is medially situated, between the cerebral hemispheres, and anterior-inferiorly bounded to the hypothalamus.

\section{FIGUERE LEGENDS:}

Figure 1. General overview of brain glucose-sensing mechanisms. (A) Hypothalamic, brainstem and corticolimbic structures contain glucose-sensing neurons that can be classified as GE or GI. (B) High glucose levels depolarize GE neurons via GK and ATPmediated closure of $\mathrm{K}_{\mathrm{ATP}}$ channels. ROS generation may also act as a signaling molecule implicated in glucose-sensing. In contrast, low glucose levels activate GI neurons via a mechanism that involves AMPK, NO and closure of chloride channels.

Figure 2. Hypothalamic control of endocrine pancreatic function. (1) Specific subsets of ARC (POMC and AgRP) and VMN (SF1) neurons sense fluctuations in circulating factors, conveying information on energy status. The VMN critically initiates the counterregulatory response to hypoglycemia in part by increasing pancreatic glucagon release. ARC neurons may be preferentially involved in adjusting post-prandrial insulin secretion. (2) Multisynaptic transmission via PVN, PAG, A5, Ra to the nucleus of the tractus solitarius (NTS) in the brainstem engages pancreatic effector mechanisms. (3) As a result, pancreas endocrine hormones (insulin and glucagon) are released accordingly into circulation. Endocrine pancreas control represents a part of integral and coordinated actions in additional peripheral tissues, by which the hypothalamus contributes to whole-body glucose homeostasis maintenance. 


\section{REFERENCES:}

1. Mayer, J. (1952) The glucostatic theory of regulation of food intake and the problem of obesity. Bull New Engl Med Cent 14 (2), 43-9.

2. Oomura, Y. et al. (1964) Reciprocal Activities of the Ventromedial and Lateral Hypothalamic Areas of Cats. Science 143 (3605), 484-5.

3. Anand, B.K. et al. (1964) Activity of Single Neurons in the Hypothalamic Feeding Centers: Effect of Glucose. Am J Physiol 207, 1146-54.

4. Timper, K. and Bruning, J.C. (2017) Hypothalamic circuits regulating appetite and energy homeostasis: pathways to obesity. Dis Model Mech 10 (6), 679-689.

5. Routh, V.H. et al. (2014) Hypothalamic glucose sensing: making ends meet. Front Syst Neurosci 8, 236.

6. Fioramonti, X. et al. (2017) Recent Advances in the Cellular and Molecular Mechanisms of Hypothalamic Neuronal Glucose Detection. Front Physiol 8, 875.

7. Wang, D. et al. (2015) Whole-brain mapping of the direct inputs and axonal projections of POMC and AgRP neurons. Front Neuroanat 9, 40.

8. Shimazu, T. and Minokoshi, Y. (2017) Systemic Glucoregulation by Glucose-Sensing Neurons in the Ventromedial Hypothalamic Nucleus (VMH). J Endocr Soc 1 (5), 449459.

9. Shimazu, T. et al. (1966) Reciprocal influences of the ventromedial and lateral hypothalamic nuclei on blood glucose level and liver glycogen content. Nature 210 (5041), 1178-9.

10. Ashford, M.L. et al. (1990) Glucose-induced excitation of hypothalamic neurones is mediated by ATP-sensitive K+ channels. Pflugers Arch 415 (4), 479-83.

11. Dunn-Meynell, A.A. et al. (2002) Glucokinase is the likely mediator of glucosensing in both glucose-excited and glucose-inhibited central neurons. Diabetes 51 (7), 205665.

12. Claret, M. et al. (2007) AMPK is essential for energy homeostasis regulation and glucose sensing by POMC and AgRP neurons. J Clin Invest 117 (8), 2325-36.

13. Stanley, S. et al. (2013) Profiling of Glucose-Sensing Neurons Reveals that GHRH Neurons Are Activated by Hypoglycemia. Cell Metab 18 (4), 596-607. 
14. Ruud, J. et al. (2017) Neuronal control of peripheral insulin sensitivity and glucose metabolism. Nat Commun 8, 15259.

15. Pocai, A. et al. (2005) Central leptin acutely reverses diet-induced hepatic insulin resistance. Diabetes 54 (11), 3182-9.

16. Titchenell, P.M. et al. (2015) Hepatic insulin signalling is dispensable for suppression of glucose output by insulin in vivo. Nat Commun 6, 7078.

17. Ramnanan, C.J. et al. (2013) Interaction between the central and peripheral effects of insulin in controlling hepatic glucose metabolism in the conscious dog. Diabetes 62 (1), 74-84.

18. Levin, B.E. and Sherwin, R.S. (2011) Peripheral glucose homeostasis: does brain insulin matter? J Clin Invest 121 (9), 3392-5.

19. Rojas, J.M. and Schwartz, M.W. (2014) Control of hepatic glucose metabolism by islet and brain. Diabetes Obes Metab 16 Suppl 1, 33-40.

20. Perrin, C. et al. (2004) Intracerebroventricular infusion of glucose, insulin, and the adenosine monophosphate-activated kinase activator, 5-aminoimidazole-4carboxamide-1-beta-D-ribofuranoside, controls muscle glycogen synthesis. Endocrinology 145 (9), 4025-33.

21. Minokoshi, Y. et al. (1999) Microinjection of leptin into the ventromedial hypothalamus increases glucose uptake in peripheral tissues in rats. Diabetes 48 (2), 287-91.

22. Haque, M.S. et al. (1999) Role of the sympathetic nervous system and insulin in enhancing glucose uptake in peripheral tissues after intrahypothalamic injection of leptin in rats. Diabetes 48 (9), 1706-12.

23. Toda, C. et al. (2009) Distinct effects of leptin and a melanocortin receptor agonist injected into medial hypothalamic nuclei on glucose uptake in peripheral tissues. Diabetes 58 (12), 2757-65.

24. Coutinho, E.A. et al. (2017) Activation of SF1 Neurons in the Ventromedial Hypothalamus by DREADD Technology Increases Insulin Sensitivity in Peripheral Tissues. Diabetes 66 (9), 2372-2386.

25. Shiuchi, T. et al. (2009) Hypothalamic orexin stimulates feeding-associated glucose utilization in skeletal muscle via sympathetic nervous system. Cell Metab 10 (6), 46680. 
26. Scherer, T. et al. (2011) Brain insulin controls adipose tissue lipolysis and lipogenesis. Cell Metab 13 (2), 183-94.

27. Buettner, C. et al. (2008) Leptin controls adipose tissue lipogenesis via central, STAT3-independent mechanisms. Nat Med 14 (6), 667-75.

28. Brito, M.N. et al. (2007) Differential activation of the sympathetic innervation of adipose tissues by melanocortin receptor stimulation. Endocrinology 148 (11), 533947.

29. Nogueiras, R. et al. (2007) The central melanocortin system directly controls peripheral lipid metabolism. J Clin Invest 117 (11), 3475-88.

30. Morgan, D.A. et al. (2015) Regulation of glucose tolerance and sympathetic activity by MC4R signaling in the lateral hypothalamus. Diabetes 64 (6), 1976-87.

31. Steculorum, S.M. et al. (2016) AgRP Neurons Control Systemic Insulin Sensitivity via Myostatin Expression in Brown Adipose Tissue. Cell 165 (1), 125-38.

32. Rosario, W. et al. (2016) The Brain-to-Pancreatic Islet Neuronal Map Reveals Differential Glucose Regulation From Distinct Hypothalamic Regions. Diabetes 65 (9), 2711-23.

33. Osundiji, M.A. et al. (2012) Brain glucose sensors play a significant role in the regulation of pancreatic glucose-stimulated insulin secretion. Diabetes 61 (2), 321-8.

34. Lam, T.K. et al. (2005) Regulation of blood glucose by hypothalamic pyruvate metabolism. Science 309 (5736), 943-7.

35. Chen, M. et al. (1975) Effect of cerebral intraventricular insulin on pancreatic insulin secretion in the dog. Diabetes 24 (10), 910-4.

36. Yang, T.T. et al. (2010) Opening of ATP-sensitive potassium channel by insulin in the brain-induced insulin secretion in Wistar rats. Horm Metab Res 42 (2), 110-4.

37. Obici, S. et al. (2002) Hypothalamic insulin signaling is required for inhibition of glucose production. Nat Med 8 (12), 1376-82.

38. Fan, W. et al. (2000) The central melanocortin system can directly regulate serum insulin levels. Endocrinology 141 (9), 3072-9.

39. Savontaus, E. et al. (2004) Metabolic effects of transgenic melanocyte-stimulating hormone overexpression in lean and obese mice. Endocrinology 145 (8), 3881-91.

40. Obici, S. et al. (2001) Central melanocortin receptors regulate insulin action. J Clin Invest 108 (7), 1079-85. 
41. Pierroz, D.D. et al. (2002) Effects of acute and chronic administration of the melanocortin agonist MTII in mice with diet-induced obesity. Diabetes 51 (5), 1337-45. 42. Heijboer, A.C. et al. (2005) Intracerebroventricular administration of melanotan II increases insulin sensitivity of glucose disposal in mice. Diabetologia 48 (8), 1621-6.

43. Marks, J.L. and Waite, K. (1996) Some acute effects of intracerebroventricular neuropeptide $\mathrm{Y}$ on insulin secretion and glucose metabolism in the rat. J Neuroendocrinol 8 (7), 507-13.

44. Korner, J. et al. (2003) Effects of agouti-related protein on metabolism and hypothalamic neuropeptide gene expression. J Neuroendocrinol 15 (12), 1116-21.

45. Ramirez, S. et al. (2017) Mitochondrial Dynamics Mediated by Mitofusin 1 Is Required for POMC Neuron Glucose-Sensing and Insulin Release Control. Cell Metab 25 (6), 1390-1399 e6.

46. Vogt, M.C. et al. (2014) Neonatal insulin action impairs hypothalamic neurocircuit formation in response to maternal high-fat feeding. Cell 156 (3), 495-509.

47. Rohner-Jeanrenaud, F. and Jeanrenaud, B. (1980) Consequences of ventromedial hypothalamic lesions upon insulin and glucagon secretion by subsequently isolated perfused pancreases in the rat. J Clin Invest 65 (4), 902-10.

48. Frohman, L.A. and Bernardis, L.L. (1971) Effect of hypothalamic stimulation on plasma glucose, insulin, and glucagon levels. Am J Physiol 221 (6), 1596-603.

49. Borg, W.P. et al. (1994) Ventromedial hypothalamic lesions in rats suppress counterregulatory responses to hypoglycemia. J Clin Invest 93 (4), 1677-82.

50. Borg, M.A. et al. (1997) Local ventromedial hypothalamus glucose perfusion blocks counterregulation during systemic hypoglycemia in awake rats. J Clin Invest 99 (2), 361-5.

51. McCrimmon, R.J. et al. (2006) Activation of AMP-activated protein kinase within the ventromedial hypothalamus amplifies counterregulatory hormone responses in rats with defective counterregulation. Diabetes 55 (6), 1755-60.

52. McCrimmon, R.J. et al. (2008) Key role for AMP-activated protein kinase in the ventromedial hypothalamus in regulating counterregulatory hormone responses to acute hypoglycemia. Diabetes 57 (2), 444-50. 
53. Evans, M.L. et al. (2004) Hypothalamic ATP-sensitive $K+$ channels play a key role in sensing hypoglycemia and triggering counterregulatory epinephrine and glucagon responses. Diabetes 53 (10), 2542-51.

54. Paranjape, S.A. et al. (2010) Influence of insulin in the ventromedial hypothalamus on pancreatic glucagon secretion in vivo. Diabetes 59 (6), 1521-7.

55. Stanley, S.A. et al. (2016) Bidirectional electromagnetic control of the hypothalamus regulates feeding and metabolism. Nature 531 (7596), 647-50.

56. Steinbusch, L.K. et al. (2016) Sex-Specific Control of Fat Mass and Counterregulation by Hypothalamic Glucokinase. Diabetes 65 (10), 2920-31.

57. Miki, T. et al. (2001) ATP-sensitive K+ channels in the hypothalamus are essential for the maintenance of glucose homeostasis. Nat Neurosci 4 (5), 507-12.

58. Chan, O. et al. (2007) ATP-sensitive $K(+)$ channels regulate the release of GABA in the ventromedial hypothalamus during hypoglycemia. Diabetes 56 (4), 1120-6.

59. Chan, O. et al. (2008) Increased GABAergic tone in the ventromedial hypothalamus contributes to suppression of counterregulatory responses after antecedent hypoglycemia. Diabetes 57 (5), 1363-70.

60. Tong, Q. et al. (2007) Synaptic glutamate release by ventromedial hypothalamic neurons is part of the neurocircuitry that prevents hypoglycemia. Cell Metab 5 (5), 383-93.

61. Meek, T.H. et al. (2016) Functional identification of a neurocircuit regulating blood glucose. Proc Natl Acad Sci U S A 113 (14), E2073-82.

62. Klockener, T. et al. (2011) High-fat feeding promotes obesity via insulin receptor/PI3K-dependent inhibition of SF-1 VMH neurons. Nat Neurosci 14 (7), 911-8.

63. Dhillon, H. et al. (2006) Leptin directly activates SF1 neurons in the VMH, and this action by leptin is required for normal body-weight homeostasis. Neuron 49 (2), 191203.

64. Roncero, I. et al. (2004) Expression of glucose transporter isoform GLUT-2 and glucokinase genes in human brain. J Neurochem 88 (5), 1203-10.

65. Smeets, P.A. et al. (2005) Functional MRI of human hypothalamic responses following glucose ingestion. Neuroimage 24 (2), 363-8. 
66. Heni, M. et al. (2012) Nasal insulin changes peripheral insulin sensitivity simultaneously with altered activity in homeostatic and reward-related human brain regions. Diabetologia 55 (6), 1773-82.

67. Hallschmid, M. et al. (2004) Intranasal insulin reduces body fat in men but not in women. Diabetes 53 (11), 3024-9.

68. Iwen, K.A. et al. (2014) Intranasal insulin suppresses systemic but not subcutaneous lipolysis in healthy humans. J Clin Endocrinol Metab 99 (2), E246-51.

69. Gancheva, S. et al. (2015) Effects of intranasal insulin on hepatic fat accumulation and energy metabolism in humans. Diabetes 64 (6), 1966-75.

70. Benedict, C. et al. (2011) Intranasal insulin enhances postprandial thermogenesis and lowers postprandial serum insulin levels in healthy men. Diabetes 60 (1), 114-8.

71. Heni, M. et al. (2014) Central insulin administration improves whole-body insulin sensitivity via hypothalamus and parasympathetic outputs in men. Diabetes 63 (12), 4083-8.

72. Heni, M. et al. (2017) Hypothalamic and Striatal Insulin Action Suppresses Endogenous Glucose Production and May Stimulate Glucose Uptake During Hyperinsulinemia in Lean but Not in Overweight Men. Diabetes 66 (7), 1797-1806.

73. Born, J. et al. (2002) Sniffing neuropeptides: a transnasal approach to the human brain. Nat Neurosci 5 (6), 514-6.

74. Kishore, P. et al. (2011) Activation of K(ATP) channels suppresses glucose production in humans. J Clin Invest 121 (12), 4916-20.

75. Esterson, Y.B. et al. (2016) Central Regulation of Glucose Production May Be Impaired in Type 2 Diabetes. Diabetes 65 (9), 2569-79.

76. Kullmann, S. et al. (2015) Selective insulin resistance in homeostatic and cognitive control brain areas in overweight and obese adults. Diabetes Care 38 (6), 1044-50.

77. Schwartz, M.W. et al. (2013) Cooperation between brain and islet in glucose homeostasis and diabetes. Nature 503 (7474), 59-66.

78. Freire-Regatillo, A. et al. (2017) Non-Neuronal Cells in the Hypothalamic Adaptation to Metabolic Signals. Front Endocrinol (Lausanne) 8, 51.

79. Young, J.K. et al. (2000) The brain response to 2-deoxy glucose is blocked by a glial drug. Pharmacol Biochem Behav 67 (2), 233-9. 
80. Chari, M. et al. (2011) Glucose transporter-1 in the hypothalamic glial cells mediates glucose sensing to regulate glucose production in vivo. Diabetes 60 (7), 19016.

81. Marty, N. et al. (2005) Regulation of glucagon secretion by glucose transporter type 2 (glut2) and astrocyte-dependent glucose sensors. J Clin Invest 115 (12), 3545-53.

82. Garcia-Caceres, C. et al. (2016) Astrocytic Insulin Signaling Couples Brain Glucose Uptake with Nutrient Availability. Cell 166 (4), 867-880.

83. Steinbusch, L. et al. (2015) Brain glucose sensing in homeostatic and hedonic regulation. Trends Endocrinol Metab 26 (9), 455-66.

84. Koekkoek, L.L. et al. (2017) Glucose-Sensing in the Reward System. Front Neurosci $11,716$.

85. Garfield, A.S. et al. (2014) A parabrachial-hypothalamic cholecystokinin neurocircuit controls counterregulatory responses to hypoglycemia. Cell Metab 20 (6), 1030-7.

86. Verberne, A.J. et al. (2014) Neural pathways that control the glucose counterregulatory response. Front Neurosci 8, 38.

87. Lam, C.K. et al. (2011) Hypothalamic nutrient sensing activates a forebrainhindbrain neuronal circuit to regulate glucose production in vivo. Diabetes 60 (1), 10713.

88. Zhang, Y. et al. (1994) Positional cloning of the mouse obese gene and its human homologue. Nature 372 (6505), 425-32.

89. Pelleymounter, M.A. et al. (1995) Effects of the obese gene product on body weight regulation in ob/ob mice. Science 269 (5223), 540-3.

90. Schwartz, M.W. et al. (1996) Specificity of leptin action on elevated blood glucose levels and hypothalamic neuropeptide $Y$ gene expression in ob/ob mice. Diabetes 45 (4), 531-5.

91. Hidaka, S. et al. (2002) Chronic central leptin infusion restores hyperglycemia independent of food intake and insulin level in streptozotocin-induced diabetic rats. FASEB J 16 (6), 509-18.

92. Coppari, R. et al. (2005) The hypothalamic arcuate nucleus: a key site for mediating leptin's effects on glucose homeostasis and locomotor activity. Cell Metab 1 (1), 63-72. 
93. Kamohara, S. et al. (1997) Acute stimulation of glucose metabolism in mice by leptin treatment. Nature 389 (6649), 374-7.

94. Yu, X. et al. (2008) Making insulin-deficient type 1 diabetic rodents thrive without insulin. Proc Natl Acad Sci U S A 105 (37), 14070-5.

95. Fujikawa, T. et al. (2010) Leptin therapy improves insulin-deficient type 1 diabetes by CNS-dependent mechanisms in mice. Proc Natl Acad Sci U S A 107 (40), 17391-6.

96. Fujikawa, T. et al. (2013) Leptin engages a hypothalamic neurocircuitry to permit survival in the absence of insulin. Cell Metab 18 (3), 431-44. 


\section{Outstanding Questions}

- How heterogeneous are glucose-sensing neurons and molecular mechanisms?

- What are the precise neurocircuits linking glucose-sensing information with effector systems?

- How multiple glucose-sensing brain regions cooperate? Is there also a crosstalk with peripheral sensors to precisely maintain euglycemia?

- Does the hypothalamus play a relevant role in the regulation of glucose homeostasis in humans? Powerful non-invasive technical advances are needed to investigate this in a reliable and comprehensive manner.

- What is the actual contribution of the hypothalamus in ensuring adequate glucoregulation under normal physiology and meal-scale? 
Figure 1

A.

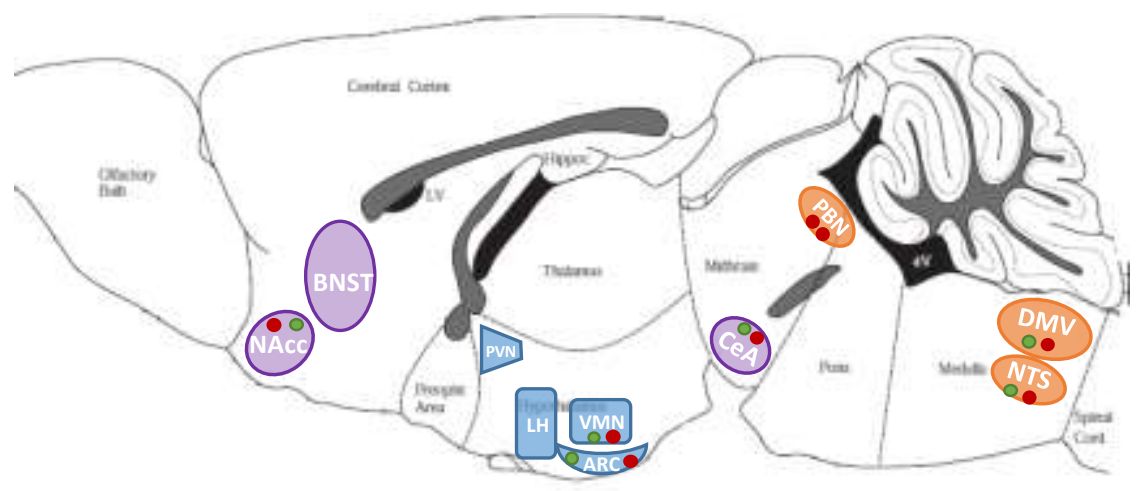

B.

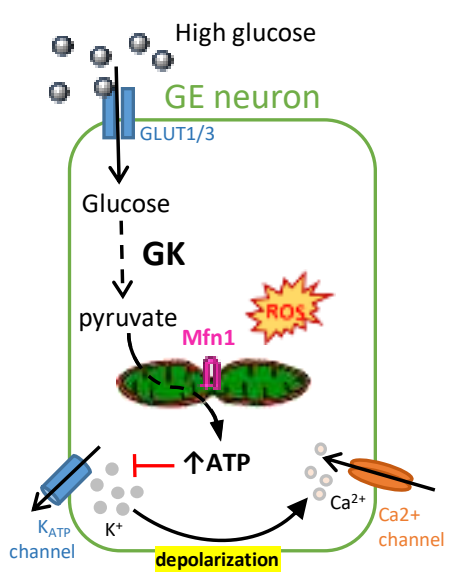

i.e. POMC neuron

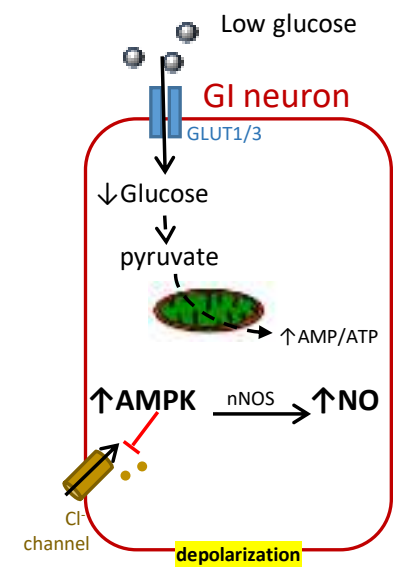

i.e. SF1 neuron 
Figure 2

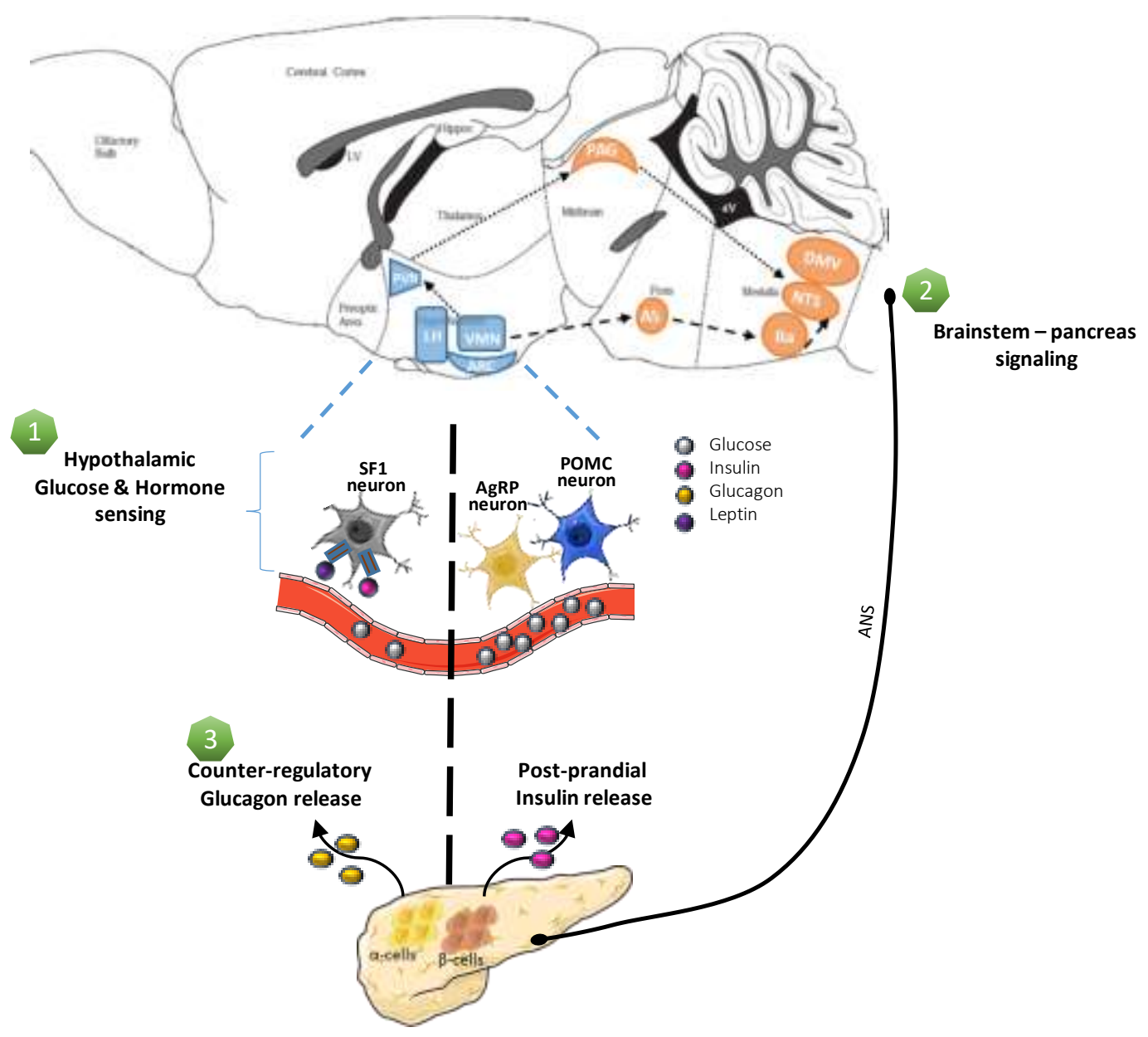

